\title{
Potential Effect of Steroidal Alkaloids from Sarcococca saligna against Leishmania tropica
}

\author{
Naeem Ullah Jan ${ }^{1, *}$, Bashir Ahmad², Muhammad Kifayatullah', Haroon Rahim¹, Fazli Amin ${ }^{1}$ \\ ${ }^{1}$ Department of Pharmacy, Sarhad University of Science and Information Technology, Peshawar, PAKISTAN. \\ ${ }^{2}$ Centre of Biotechnology and Microbiology, University of Peshawar, Peshawar, PAKISTAN.
}

\begin{abstract}
Background and Objectives: Sarcococca species have a rich source of steroidal alkaloids that possess different biological and pharmacological activities. Hence, present research was design to isolate the steroidal alkaloid from Sarcococca saligna and to evaluate it for in-vitro antileishmanial activity. Materials and Methods: Compound Holaphylline and Saracodine were isolated through column chromatography followed by spectroscopic techniques for elucidation and were assayed against promastigotes of Leishmania tropica. Results: Saracodine compound eliminates $71.12 \%, 63.9 \%, 54.18 \%$ and $38.88 \%$ of the promastigotes at concentration $100 \mu \mathrm{M} ; 75 \mu \mathrm{M}, 50 \mu \mathrm{M}$ and $(25 \mu \mathrm{M})$. While, the elimination of promastigotes with compound holaphylline were $82.5 \%, 76.68 \%, 65 \%$, $47.22 \%$ respectively as compared to untreated group. Conclusion: In present study, compound Holaphylline and Saracodine exhibited inhibition against the promastigotes and justified the claimed medicinal importance of Sarcococca saligna as a remedy for leishmaniasis especially against Leishmania tropica.
\end{abstract}

Key words: Sarcococca saligna, Holaphylline, Saracodine, Leishmania tropica, Promastigotes, Chloroform extract.

\section{INTRODUCTION}

The genus Leishmania is a protozoan parasite that is mainly responsible for a group of leishmaniasis diseases in mammal and almost affects 15 million of worldwide population particularly children followed by young adults. ${ }^{1}$ L. donovani, $L$. amazonesis, $L$. maxicana, L. chagasi, L. tropica are the main types of leishmania species. ${ }^{2}$ This disease is being claimed as one of the most public health care burden in subtropical and tropical region of Africa, Asia, Central and South America and Mediterranean regions. ${ }^{2}$ Liver, bone marrow, spleen and lymph nodes are the main organs affected with these parasites and if left untreated might be fatal as reported in literature. ${ }^{3}$ Proven treatments against leishmaniasis include stibamine, sodium stibogluconate and urea that are not so effective due to undesirable effects and limited efficacy. ${ }^{4}$ Additionally, Amphotericin- $\mathrm{B}$ and pentamidine are the common allopathic drugs in treating leishmaniasis, but their prolonged uses may not be beneficial. Therefore, in this case there is an urged to develop a new and more effective remedy from natural products with antiprotozoal activity against leishmaniasis having no harmful effect with maximum benefits. Plant based medicines are being claimed as one of the important source for the development of drug in treating leishmaniasis diseases. ${ }^{5}$ Many research studies showed that many plants extract and thire derived possess anti-leishamanial activity against different species of Leishmania. ${ }^{5}$ In the same way one of the plant belongs to Sarcococca species of Buxaceae family have rich source of steroidal alkaloids that possess different biological activities in endemic region of Pakistan. ${ }^{6}$ Sarcococca saligna (D.Don) Muel belongs to family of Buxaceae, that exist in the northern areas of Pakistan. The shoots
Submission Date: 09-07-2019; Revision Date: 27-09-2019; Accepted Date: 04-10-2019

DOI: 10.5530/ijper.53.4s.161 Correspondence: Dr. Naeem Ullah Jan, Department of Pharmacy, Faculty Life Sciences, Sarhad University of Science and Information Technology, Peshawar, Khyber-Pakhtunkhwa, PAKISTAN.

Phone: +923337489718 E-mail: numan2001@ hotmail. com

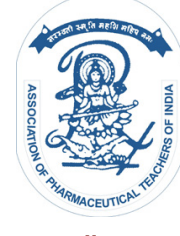

www.ijper.org 
and leaves of Sarcococca saligna are being reported for having significant effects against muscles pain, stomach problem and disorder of blood. ${ }^{7}$ Steroidal alkaloids isolated from various other species of Sarcococca are scientifically proven for different biological and pharmacological activities. The extract fractions of Sarcococca saligna was reported in literature for significant potential against skin infections, malaria, muscle pain and rheumatism. ${ }^{7}$ Previous bioactive steroidal alkaloids isolated from Sarcococca saligna have documented for strong antidiarrhoeal as well as hypersecretion effect in mice. ${ }^{8}$ Different alkaloids from natural product have been demonstrated for possessing antileishmanial, antibacterial and potent acetylcholinestrase inhibitors. ${ }^{7}$ Research associated with steroidal alkaloids of Sarcococca species has been showed hepatoprotective potential experiementlly. ${ }^{9}$ So, keeping in view of established importance of Sarcococa saligna it has been further explored for antileishmanial activity. Therefore, the goal of this research paper was to isolate compounds from Sarcococca saligna and to screen it for in-vitro antileshmanial activity.

\section{MATERIALS AND METHODS}

\section{Collection and Identification of Plant Material}

The whole plant "Sarcococca saligna”(D.Don) Muel (40 kg) was collected in June, 2014 from District Swat, Khyber Pukhtunkhwa, Pakistan. The identification and authentication was done by botanist Dr. Jilani with specimen vide BUT-20098 (pup) was deposited to Department of Botany (herbarium section), University of Peshawar, Pakistan.

\section{Chemicals and Instruments}

Column chromatography techniques with various analytical grade reagents along silica gel $\left(\mathrm{SiO}_{2}\right)$ and alumina $\left(\mathrm{Al}_{2} \mathrm{O}_{3}\right)$ were used as a stationary phase. TLC plates (Merck GF-254) followed by (pre-coated) $\mathrm{SiO}_{2}$ and Dragendroff's reagent were used for the visualization of alkaloids. Mass spectrometer (Jeol HX-110), IR Jasco A-302, Bruker Avance AM-400 and AC-300 NMR and Hitachi UV-3200 spectrophotometer were used for structure determination of compounds.

\section{Extraction and Fractionation}

Sarcococca saligna was shade dried for a period of 03 weeks and crushed in to coarse powder with the help of mechanical blender. The crude drug was macerated in hydromethanolic mixture with 8:2 ratios in $35 \mathrm{~L}$ for a period of two weeks at normal room temperature with intermittent shaking. The mixture was first filtered and then concentrated $(2 \mathrm{~kg})$ under vacuum. Two liters of distilled water was poured in concentrated $\mathrm{MeOH} / \mathrm{H}_{2} \mathrm{O}$ extract. The mixtures $(\mathrm{MeOH} / \mathrm{H} 2 \mathrm{O}$ extract) was again extracted through different solvent system starting from $\mathrm{n}$-hexane followed by chloroform, ethyl acetate and butanol and get different fractions i.e. n-hexane (254 $\mathrm{g})$, chloroform (200g), ethyl acetate (150g) and butanol $(100 \mathrm{~g}) \cdot{ }^{10}$

After pilot study on each fraction against Leishmania tropica, the chloroform fraction was selected based on potent activity and was subjected further for detection of alkaloids by using Dragendroff's spray on TLC. For isolation and purification of alkaloids, chloroform fraction was subjected further to column chromatography starting from n-hexane and ethyl acetate solvent system in different ratio along with few drops of diethylamine. Different sub fractions F1 to F3 were attained by subjecting to alumina gel column chromatography for purification, eluted with different ratio of n-hexane followed by ethyl acetate and some drops of diethylamine. ${ }^{11}$ Holaphylline $(135 \mathrm{mg})$ and Saracodine (125 $\mathrm{mg}$ ) was attained from fraction (F3) by increasing the polarities of $n$-hexane/ethyl acetate followed with some diethyl amine drops in column chromatography. The Holaphylline $(135 \mathrm{mg})$ and Saracodine $(125 \mathrm{mg})$ that was collected from fraction (F3) was screened for antileishmanial Activity.

\section{Holaphylline}

Holaphylline appear as sticky yellowish light powder. The M.P. is $125^{\circ} \mathrm{C}$ (Reported $128^{\circ} \mathrm{C}$ ). FD-MS: $\mathrm{m} / \mathrm{z}$ 329.27 (C22H35NO). HREI-MS m/z: 329.55 (calcd, $\left.\mathrm{C}_{22} \mathrm{H}_{35} \mathrm{NO}, 329.4529\right)$. ${ }^{1} \mathrm{H}-\mathrm{NMR}\left(\mathrm{CDCl}_{3}\right): \delta 0.90(3 \mathrm{H}$, s, $\left.\mathrm{CH}_{3}-18\right), 1.00\left(3 \mathrm{H}, \mathrm{s}, \mathrm{CH}_{3}-19\right), 2.10\left(3 \mathrm{H}, \mathrm{s}, \mathrm{CH}_{3}-21\right)$, $2.62\left(3 \mathrm{H}, \mathrm{s}, \mathrm{CH}_{3}-22\right), 2.99$ (1 H,dd,H-3 ),5.34(1H,brs, $\mathrm{H}-6), 2.78(1 \mathrm{H}, \mathrm{brs}, \mathrm{H}-17) .{ }^{13} \mathrm{C}-\mathrm{NMR}\left(\mathrm{CDCl}_{3}\right) ; \delta=35.0$ (C-1), 25.9(C-2), 59.2(C-3), 37.0(C-4), 137.7(C-5), 123.6(C-6), 31.9(C-7), 31.8(C-8), 49.3(C-9), 36.7(C-10), 20.9(C-11), 34.9(C-12),45.4(C-13), 50.4(C-14), 24.3(C15), 25.1(C-16), 61.2(C-17),20.6(C-18), $19.20(\mathrm{C}-19)$, 212.7(C-20), 32.8( C-21), 29.9(C-22). The spectral data indicated that holaphylline was well known in research but isolated for first time from the species of Sarcococca saligna and previously reported their isolation from $\mathrm{Hol}$ arrbena floribunda plant. $^{12}$

\section{Structure of Holaphylline}

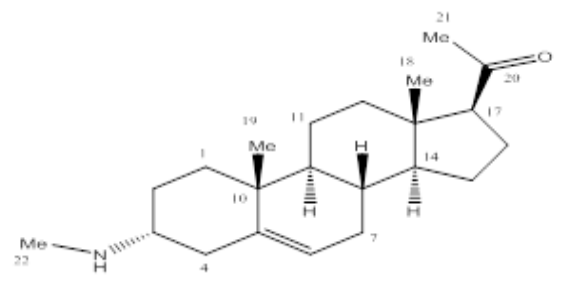


Mass Spectra of Holaphylline

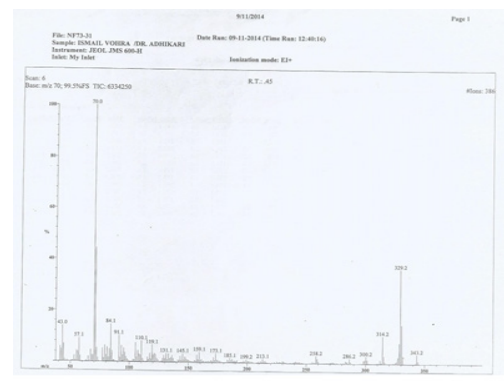

${ }^{1}$ HNMR of Holaphyline

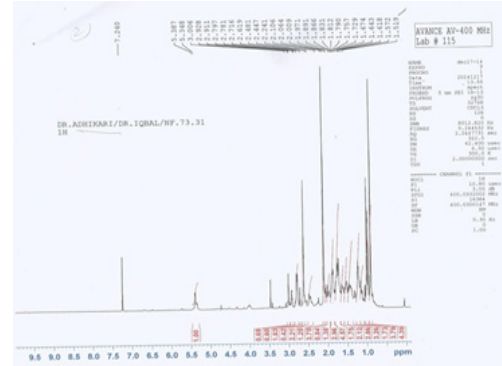

\section{Saracodine}

The physical appearances of this compound occur as white amorphous powder. M.P is $242-244^{\circ} \mathrm{C}$, [Reported value, $244-246^{\circ} \mathrm{C}$. EI MS m/z (rel.int \% ): 402 ( 29 ), $\left[\mathrm{M}^{+}\right], 387$ ( 2) [ $\left.\mathrm{M}^{+}-15\right], 110$ ( 62 ), 100 ( 15 ), 84 ( 100), 72 ( 22). FD-MS: $m / z 402\left(\mathrm{C}_{26} \mathrm{H}_{46} \mathrm{~N}_{2} \mathrm{O}\right)$. HREI-MS $\mathrm{m} / \mathrm{z}: 402.2579$ (calcd, $\mathrm{C}_{26} \mathrm{H}_{46} \mathrm{~N}_{2} \mathrm{O}, 402.2609$ ). ${ }^{1} \mathrm{H}-\mathrm{NMR}$ $\left(\mathrm{CDCl}_{3}\right): \delta 0.69 / 0.72\left(3 \mathrm{H}, \mathrm{s}, \mathrm{CH}_{3}-18\right), 0.79 / 0.80(3 \mathrm{H}$, $\left.\mathrm{s}, \mathrm{CH}_{3}-19\right), 1.04 / 1.14\left(3 \mathrm{H}, \mathrm{d}, J_{20,21}=6.5 \mathrm{~Hz}, \mathrm{CH}_{3}-\right.$ 21), 2.01/2.07 (3H, s, $\left.\mathrm{COCH}_{3}\right), 2.21\left(6 \mathrm{H}, \mathrm{s}, \mathrm{N}\left(\mathrm{CH}_{3}\right)_{2}\right)$, 2.71/2.75 (3H, s, $\left.\mathrm{N}_{b}-\mathrm{CH}_{3}\right), 3.58 / 4.61(1 \mathrm{H}, \mathrm{m}, \mathrm{H}-20)$. The spectral data shows that saracodine was isolated previously from the species of Sarcococca saligna. ${ }^{13}$

\section{Structure of Saracodine}

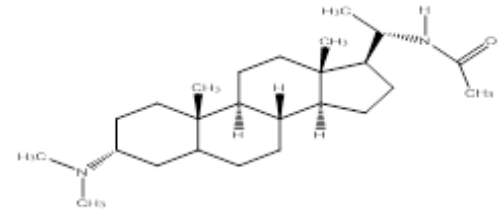

Mass Spectra of Saracodine

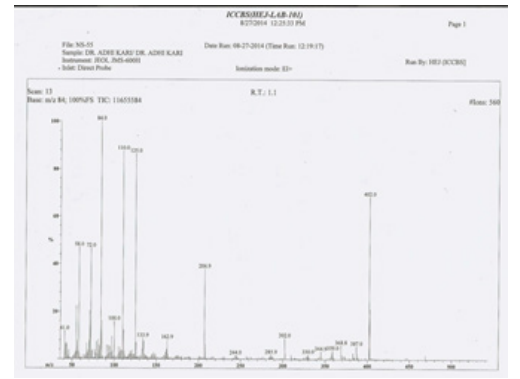

${ }^{1}$ HNMR of Sarcodine

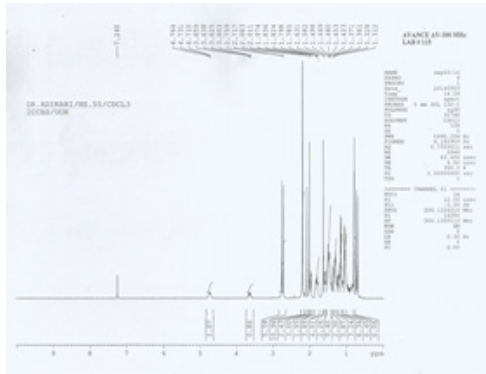

\section{Anti-leishmanial Activity}

\section{Parasite Culture}

Leishmania tropica was cultured in the Department of Zoology, University of Peshawar of cryopreserved culture at Molecular and Culture Laboratory. First the culture was produced in 5mL M-199 medium supplemented with $10 \%$ heat-inactivated fetal bovine serum (HI-FBS), $100 \mu \mathrm{g} / \mathrm{mL}$ penicillin, $50 \mu \mathrm{g} / \mathrm{mL}$ kanamycin, $100 \mu \mathrm{g} / \mathrm{mL}$ streptomycin and $5 \mu \mathrm{g} / \mathrm{mL}$ of Hemin in 15 $\mathrm{mL}$ culture flask and then incubated at $26^{\circ} \mathrm{C}$. Then the medium was changed after third day. For development of promastigoes into metacyclic stage, the cultures were placed for period of 10 to 14 days followed by suspending the promastigoes for centrifugation at $2000 \mathrm{rpm}$ for $12 \mathrm{~min}$. The medium was spill off leaving the pellets only. These pellets were suspended again in 2 to $4 \mathrm{~mL}$ growth medium and new cultures were developed. ${ }^{14}$

\section{Anti-Promastigote Potential for isolated Compounds}

The isolated compounds (Holaphylline, Saracodine) were evaluated for anti-leishmanial activity. From the viable promastigotes, bulk culture $\left(3.7 \times 10^{7} / \mathrm{mL}\right), 1 \times 10^{5}$ promastigotes/well in $200 \mu \mathrm{L}$ fresh M-199 medium were seeded in 96-well plate. Four concentrations of the compounds were prepared and the control was placed in one row of 12 wells having only the growth medium. The 96 wells plates were incubated at $26^{\circ} \mathrm{C}$ for $48 \mathrm{hr}$ and Improved Neubauer Haemocytometer was used for counting the promastigotes in each well of both treated, control.

The $\%$ inhibition of promastigotes was calculated by the formula:

$\%$ inhibition $=$ No. of control promastigotes- No. of treated promastigotes ${ }^{15,16}$ No. of control promastigotes

\section{RESULTS AND DISCUSSION}

Present research work examined the anti-promastigote potential of both compounds (Holaphylline, Saracodine) against Leishmania tropica that were isolated from Sarco- 
Table 1: Anti-leishmanial activity of Saracodine and Holaphylline against promastigotes of $L$. tropica.

\begin{tabular}{|c|c|c|c|}
\hline $\begin{array}{l}\text { Tested } \\
\text { compound }\end{array}$ & $\begin{array}{l}\text { Different } \\
\text { conc. in } \mu \mathrm{M}\end{array}$ & $\begin{array}{c}\text { Mean } \% \text { Inhibition } \\
\pm \text { SD }\end{array}$ & $\mathrm{IC}_{50}(\mu \mathrm{M})$ \\
\hline Holaphylline & 100 & $82.5 \pm 4.83$ & \multirow{4}{*}{0.6675} \\
\hline Holaphylline & 75 & $76.68 \pm 7.87$ & \\
\hline Holaphylline & 50 & $65.0 \pm 2.96$ & \\
\hline Holaphylline & 25 & $47.22 \pm 2.65$ & \\
\hline Saracodine & 100 & $71.12 \pm 5.20$ & \multirow{4}{*}{0.6352} \\
\hline Saracodine & 75 & $63.9 \pm 8.41$ & \\
\hline Saracodine & 50 & $54.18 \pm 8.69$ & \\
\hline Saracodine & 25 & $38.88 \pm 5.98$ & \\
\hline Pentamidine* & 100 & $63.5 \pm 6.09$ & \multirow{4}{*}{0.0199} \\
\hline Pentamidine* & 75 & $57.5 \pm 7.54$ & \\
\hline Pentamidine* & 50 & $52.5 \pm 9.65$ & \\
\hline Pentamidine* & 25 & $33.75 \pm 5.81$ & \\
\hline
\end{tabular}

cocca saligna. The $\%$ inhibitions of both compounds were measured by comparing the treated group to untreated group. After 48 hrs of the treatment the average number of promastigotes was 90.00 in control group. The $\mathrm{IC}_{50}$ of the isolated compounds was calculated with the help of Graph Pad Prism 6 Software along with 95\% confidence intervals

\section{Anti-Promastigote Activity of Compound Saracodine}

Table 1 presents the anti-leishmanial activity of Saracodine against Leishmania tropica. The promastigotes were treated with different concentrations $(100 \mu \mathrm{M}, 75 \mu \mathrm{M}$, $50 \mu \mathrm{M}$ and $25 \mu \mathrm{M}$ ) of Saracodine for a period of 48 hrs at $26^{\circ} \mathrm{C}$. A concentrations dependent elimination of promastigotes was observed in treated groups as compared to untreated. As clearly shown in Table 1 that Saracodine compound caused $71.12 \%$ of the promastigotes elimination at $100 \mu \mathrm{M}$ concentrations, $63.9 \%$ at $75 \mu \mathrm{M}$ concentrations, $54.18 \%$ at $50 \mu \mathrm{M}$ concentrations and the elimination of promastigotes at lowest concentration $(25 \mu \mathrm{M})$ were $38.88 \%$ as compared to untreated group. Previous research from literature review has confirmed the death or elimination of promastigote with plant metabolites because of interaction with mitochondrial membranes that leads apoptosis (mechanism similar to metazoan apoptosis). ${ }^{17}$ So, it is assumed that the steroidal alkaloid Saracodine isolated from Sarcococca saligna also presents a similar mechanism for the elimination of promastigotes. In addition with above stated mechanism, the antileishmanial effects of Saracodine might cause an enhancement in production of nitric oxide, which results in fragmentation of nuclear DNA that play an important role in death of intracellular amastigotes as previously reported in literature for other secondary metabolites of natural products. ${ }^{18}$ Although, the pharmacological data regarding the antileishmanial activity of Sarcococca saligna has not been documented until now in the literature, but the isolated steroidal alkaloids Saracodine from Sarcococca saligna possess antileishmanial activity that may be attributed to their ability for intercalation DNA as it was previously reported in literature. ${ }^{19}$ Previous research studies demonstrate the potential effect of plant based medicine in activation of macrophage that provide base in controlling of leishmania. ${ }^{20}$ So, it is assumed that the steroidal alkaloid Saracodine enhanced the elimination of promastigote due to activation of macrophage

Anti-Promastigote Activity of Compound Holaphylline Table 1 also shows the anti-leishmanial activity of Holaphylline that were isolated from Sarcococca saligna against the promastigotes of L. tropica. The cultured promastigotes were treated with same concentrations $(100 \mu \mathrm{M}, 75 \mu \mathrm{M}, 50 \mu \mathrm{M}$ and $25 \mu \mathrm{M})$ of Holaphylline for a period of $48 \mathrm{hr}$ at $26^{\circ} \mathrm{C}$. The elimination promastigotes were $82.5 \%$ at $100 \mu \mathrm{M}$ concentration, $76.68 \%$ at $75 \mu \mathrm{M}$ concentration, $65.0 \%$ at $50 \mu \mathrm{M}$ concentration followed by $47.22 \%$ at concentration $25 \mu \mathrm{M}$. Previous research studies demonstrate the potential effect of plant based medicine in activation of macrophage that provide base in controlling of leishmania disease. So, it is suggested that the steroidal alkaloid Holaphylline increased the elimination promastigotes due to activation of macrophage. ${ }^{21}$ The exact mechanism behind the direct antileishmanial effect exhibited by the Saracodine and Holaphylline is unknown, but some other researchers have hypothesized generation of free radicals by active molecule of natural products that act on parasitic DNA and thus causing elimination of promastigote. ${ }^{22}$

\section{CONCLUSION}

The results of the present study showed significant effect of Holaphylline and Saracodine against L. tropica that was isolated Sarcococca saligna. To our knowledge, this research work for the first time established the antileishmanial activity of steroidal alkaloids isolated from Sarcococca saligna.

\section{ACKNOWLEDGEMENT}

We would like to thank Prof. Dr. Bashir Ahmad, Centre of Biotechnology and Microbiology, University of Peshawar for facilitating in conducting of this research work. 


\section{CONFLICT OF INTEREST}

The authors declare no conflict of interest.

\section{ABBREVIATIONS}

TLC: Thin Layer Chromatography; IC $_{50}$ : Half Maximal Inhibitory Concentration; $\mu \mathrm{M}$ : Micromole; DNA Deoxyribonucleic Acid; NMR: Nuclear Magnetic Resonance; F1: Fraction 1; F3: Fraction 3.

\section{REFERENCES}

1. Reithinger R, Dujardin JC, Louzir H, Pirmez C, Alexander B, Brooker S. Cutaneous leishmanias. Lan Inf Dis. 2007;7(9):581-96.

2. Torres-Guerrero E, Romano MQC, Ruiz-Esmenjaud J. Leishmaniasis: A review. F1000 Res. 2017;6-10.

3. Murray HW. Advances in leishmaniasis. Lancet. 2005;366(9496):1561-77.

4. Kam TS, Sim KM, Koyano T, Toyoshima M, Hayash, M, Komiyama K. Cytotoxic and leishmanicidal aminoglycosteroids and aminosteroids from Holarrhena curtisii. J Nat Prod. 1998;61(11):1332-6.

5. Funari CS, Almeida LD, Passalacqua TG, Martinez I, Ambrósio DL. Oleanonic acid from Lippia lupulina (Verbenaceae) shows strong in vitro antileishmanial and antitrypanosomal activity. Acta Amaz. 2016;46(4):411-6.

6. Jan NU, Ahmad B, Ali S, Adhikari A, Ali A, Jahan A, et al. Evaluation of antidiabetic potential of steroidal alkaloid of Sarcococca saligna. Biomed Pharmacother. 2018;100:461-6.

7. Feroz F, Haq ZU, Feroz F, Khalid A, Nawaz SA, Khan MR, et al. New cholinesterase-inhibiting steroidal alkaloids from Sarcococca saligna. Helv Chim Acta. 2004;87(2):439-48.

8. Gilani A, Ghayur MN, Khalid A, Haq ZU, Choudhary MI, Rahman AU. Presence of antispasmodic, antidiarrheal, antisecretory, calcium antagonist and acetylcholinesterase inhibitory steroidal alkaloids in Sarcococca saligna. Planta Med. 2005;71(02):120-5.

9. Ali H, Musharraf SG, Iqbal N, Adhikari A, Abdalla OM, Ahmed MM, et al. Immunosuppressive and hepatoprotective potential of Sarcococca saligna and its biomarker components. Int Immunopharmacol. 2015;28(1):235-43.
10. Zhu $F$, Zhao L, Zhang Z, Jianget $H$. Preliminary study at lipids extraction technology from municipal sludge by organic solvent. Procedia Environ Sci. 2012;16:352-6.

11. Jan NU, Ahmad B, Ali S, Adhikari A, Ali A, Jahan A, et al. Steroidal alkaloids as an emerging therapeutic alternative for investigation of their immunosuppressive and hepatoprotective potential. Front Pharmacol. 2017;8:114-20.

12. Janot M, Cave A, Goutarel R. Sterol alkaloids. III. Togholamine, holaphyllamine and holaphylline, new alkaloids from the leaves of Holarrhena floribunda. Bull Soc Chim Fr. 1959;896-900.

13. Naeem I, Khan N, Choudhary MI. Alkaloids of Sarcococca saligna. Phytochemistry. 1996;43(4):903-6.

14. Ahmed NH. Cultivation of parasites. Trop Med Parasitol. 2014;4(2):80-9.

15. Habtemariam S. In vitro antileishmanial effects of antibacterial diterpenes from two Ethiopian Premna species: P. schimperi and P. oligotricha. BMC Clin Pharmacol. 2003;3(1):6-10.

16. Lima NM, Correia CS, Leon LL, Machado GM. Antileishmanial activity of lapachol analogues. Mem Inst Oswaldo Cruz. 2004;99(7):757-61.

17. Dutta A, Bandyopadhyay S, Mandal C, Chatterjee M. Aloe vera leaf exudate induces a caspase-independent cell death in Leishmania donovani promastigotes. J Med Microbiol. 2007;56(5):629-36.

18. Dutta A, Sarkar D, Gurib-Fakim A, Mandal C, Chatterjee M. In vitro and in vivo activity of $A$ loe vera leaf exudate in experimental visceral leishmaniasis. Parasitol Res. 2008;102(6):1235-42.

19. Braga FG, Bouzada ML, Fabri RL, OMatos DM, Moreira FO, Scio E, et al. Antileishmanial and antifungal activity of plants used in traditional medicine in Brazil. J Ethnopharmacol. 2007;111(2):396-402.

20. Cruz GV, Pereira PV, Patrício FJ, Costa GC, Sousa SM, Frazão JB, et al. Increase of cellular recruitment, phagocytosis ability and nitric oxide production induced by hydroalcoholic extract from Chenopodium ambrosioides leaves. J Ethnopharmacol. 2007;111(1):148-54

21. Montalvo AM, Almanonni S, Scull R, Miranda M, Abreu J. Activity of the essential oil from Chenopodium ambrosioides grown in Cuba against Leishmania amazonensis. Chemotherapy. 2006;52(3):130-6.

22. Monzote L, Montalvo AM, Scull R, Miranda M, Abreu J. Combined effect of the essential oil from Chenopodium ambrosioides and antileishmanial drugs on promastigotes of Leishmania amazonensis. Revista do Instituto de Medicina Tropical de São Paulo. 2007;49(4):257-60.

\section{PICTORIAL ABSTRACT}

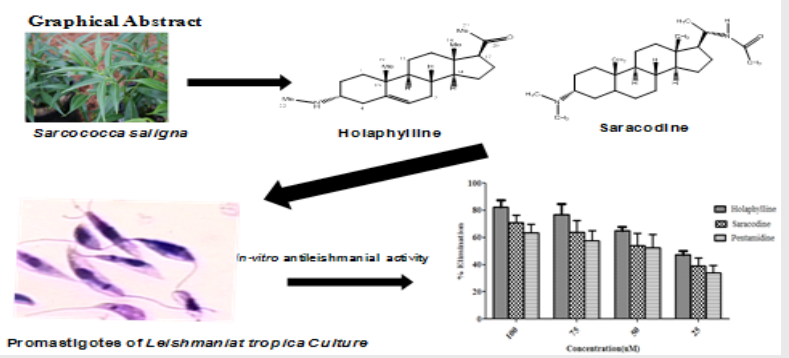

\section{SUMMARY}

Present research work showed that compounds Holaphylline and Saracodine isolated from Sarcococca saligna exhibits significant inhibition against the promastigotes of L. tropica to support their traditional used. However, comprehensive research works are needed in future to determine in-vivo studies and exact mechanism of the antileishmanial effect of both compounds that will lead to develop some potent antileishmanial drugs.

\section{About Authors}

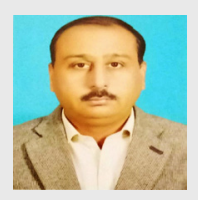

Dr. Naeem Ullah Jan, Assistant Professor, Department of Pharmacy, Sarhad University of Science and Information Technology, Peshawar, Pakistan.

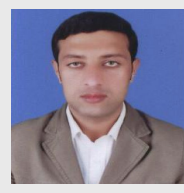

Dr. Muhammad Kifayatullah, Assistant Professor, Department of Pharmacy, Sarhad University of Science and Information Technology, Peshawar, Pakistan.

Cite this article: Jan NU, Ahmad B, Kifayatullah M, Rahim H, Amin F. Potential Effect of Steroidal Alkaloids from Sarcococca saligna against Leishmania tropica. Indian J of Pharmaceutical Education and Research. $2019 ; 53(4 s): s 650-s 654$. 